Please do not remove this page

RMIT

UNIVERSITY

\title{
Sound transmission loss of ETICS cladding systems considering the structure-borne transmission via the mechanical fixings: Numerical prediction model and experimental evaluation
}

Santoni, Andrea; Bonfiglio, Paolo; Davy, John; Fausti, Patrizio; Pompoli, Francesco; Pagnoncelli, Luigi https://researchrepository.rmit.edu.au/esploro/outputs/9921861764301341/filesAndLinks?institution=61RMIT_INST\&index=null

Santoni, A., Bonfiglio, P., Davy, J., Fausti, P., Pompoli, F., \& Pagnoncelli, L. (2017). Sound transmission loss of ETICS cladding systems considering the structure-borne transmission via the mechanical fixings: Numerical prediction model and experimental evaluation. Applied Acoustics, 122, 88-97. https://doi.org/10.1016/j.apacoust.2017.02.008

Document Version: Accepted Manuscript

Published Version: https://doi.org/10.1016/j.apacoust.2017.02.008

Repository homepage: https://researchrepository.rmit.edu.au (C) 2017 Elsevier Ltd. All rights reserved.

Downloaded On 2023/04/27 00:33:55 +1000 
Thank you for downloading this document from the RMIT Research Repository.

The RMIT Research Repository is an open access database showcasing the research outputs of RMIT University researchers.

RMIT Research Repository: http://researchbank.rmit.edu.aul

\author{
Citation: \\ Santoni, A, Bonfiglio, P, Davy, J, Fausti, P, Pompoli, F and Pagnoncelli, L 2017, \\ 'Sound transmission loss of ETICS cladding systems considering the \\ structure-borne transmission via the mechanical fixings: Numerical prediction model \\ and experimental evaluation', Applied acoustics, vol. 122, pp. 88-97.
}

See this record in the RMIT Research Repository at:

https://researchbank.rmit.edu.au/view/rmit:42104

Version: Accepted Manuscript

\title{
Copyright Statement:
}

(c) 2017. This manuscript version is made available under the CC-BY-NC-ND 4.0 license http://creativecommons.org/licenses/by-nc-nd/4.0/

Link to Published Version:

https://dx.doi.org/10.1016/j.apacoust.2017.02.008 


\title{
Sound transmission loss of ETICS cladding systems considering the structure-borne transmission via the mechanical fixings: Numerical prediction model and experimental evaluation.
}

\author{
Andrea Santoni $^{\mathrm{a}, *}$, Paolo Bonfiglio ${ }^{\mathrm{a}}$, John L. Davy ${ }^{\mathrm{b}}$, Patrizio Fausti ${ }^{\mathrm{a}}$, Francesco Pompoli ${ }^{\mathrm{a}}$, Luigi \\ Pagnoncellic
}

\begin{abstract}
${ }^{a}$ Engineering Department, University of Ferrara, via G. Saragat 1, 44122 Ferrara, Italy
${ }^{b}$ School of Science, RMIT University, GPO box 2476, Melbourne, Victoria 3001, Australia ${ }^{c}$ ROCKWOOL International A/S, Hovedgaden 584, DK-2640, Hedehusene, Denmark
\end{abstract}

\begin{abstract}
Nowadays it is common to increase the acoustic and thermal performance of building façades using the External Thermal Insulation Composite System (ETICS). In this paper a prediction model to evaluate the sound transmission loss provided by such systems, also considering the mechanical bridges that connect the insulating slabs to the basic wall, is presented. The model is developed within the transfer matrix method framework and it is based on a well established decoupled approach. The different formulations presented by several authors to take into account the structure-borne sound transmission through the mechanical connections in multilayer and double-plate elements are not suitable to analyse these kind of building partitions, since they are addressed only to thin plate elements and lightweight porous or fibrous materials. Unfortunately, the different layers that the ETICS consists of need a more sophisticated definition within the transfer matrix method. Therefore, a new model, developed for these specific elements, is proposed. The computation of all the terms and the necessary assumptions are accurately described and their validity is verified with experimental data. Finally, the reliability of the model is investigated by comparing the numerical results with measured transmission loss.
\end{abstract}

Keywords: structural-bridges, sound insulation, transfer matrix method, ETICS

\section{Introduction}

The façades of buildings have to fulfil many functions other than giving good architectural aesthetics. The façade represents the building's coat that provides protection from the external environment. It has to preserve the fabric of the building from meteorological events in order to guarantee its functionality over the years. Moreover, it plays an important role in the thermal and acoustic comfort of the indoor spaces. High thermal insulation performance is commonly achieved by using a façade cladding system, known as the External Thermal Insulation Composite System (ETICS). The external walls of the building are lined with insulating slabs on which

*Corresponding author

Email address: andrea.santoni@unife.it (Andrea Santoni)

Preprint submitted to Journal Applied Acoustics

February 3, 2017 
different finishings can be applied. The slabs are fixed to the fabric of the building using both mortar or special resins and mechanical fixings distributed over the surface of the basic wall. Furthermore, when a proper material is used, such as mineral wool, the system provides good sound insulation as well as good thermal properties. In this case the ETICS system can also enhance the acoustic performance required in buildings to meet the regulation requirements [1]. However, while great attention has been paid to the thermal performance over the last ten years, thanks to the EU policy for energy efficiency of buildings, only a few acoustic experimental investigations have been done [2]. Moreover, the development of proper tools to perform an accurate acoustic analysis of such systems is still lacking. These multilayer systems can be treated as double panel structures with mechanical point connections. The structural bridges link the building wall to the thin finishing layer through the insulating material. Therefore, the theoretical sound insulation that would be provided by the element without mechanical fixings would be reduced, due to the structure-borne sound transmission via the mechanical connections. In 2006, an extensive comparison between more than twenty different approaches to predict sound transmission in double panels was presented by Hongisto, [3]. Among them, only a few methods took into account the presence of structural connections between panels. By means of a decoupled approach, the problem is simplified by computing the fluid-borne transmission, through the cavity and the structure-borne path, through the connections, separately. The decoupled approach was developed by Sharp [4] and it considers the sound transmission through structural bridges by means of a correction term, calculated as a function of the sound power radiated by a point (or line) connected panel [5]. Sharp's method was modified and inserted into the transfer matrix framework by Vigran [6], who also extended the validity of the method above the critical frequency. A different version of the decoupled approach, mainly focused on line-connections for studs cavity walls, was developed by Fahy and Gardonio [7]. The possibility to consider studs with finite stiffness, a problem first analysed by Gu and Wang [8], was introduced into Fahy's model, through an equivalent mechanical compliance, by Davy $[9,10]$. Moreover, Davy recently published the full derivation of a prediction model for sound transmission via structural bridges, both for line and point connections [11]. The line-connections assumption is particularly useful to model cavity stud walls and double panel structures when the screw spacing is small compared to the bending wavelength of the wave propagating in the plate [12]. However, this is not the case for the ETICS system, which is not a frame based structure. Hence a point-connection approach is more suitable. Despite the huge variety of models that can be found in the literature, none of them seems to be suitable for the ETICS, since they have been developed for lightweight structures. Moreover, they consider only thin plates, and the cavity being either empty or filled with low-density porous or fibrous material, which can be modelled with good approximation using simplified theories. On the contrary, the ETICS is mounted on walls that need to be modelled as thick plates, and high-density fibrous materials are involved, which cannot be simply approximated as equivalent fluids, but need to be modelled as poroelastic media using Biot's theory. The lack of such a study on this kind of multilayer structures with mechanical point bridges motivated the work presented in this paper, in which a modified decoupled approach within the transfer matrix method is proposed, assuming massless infinitely stiff point connections. In the next section the basic background theory on the transfer matrix method is given and the numerical model is described. In section 3 the investigated structure is introduced and its modelling within the transfer matrix method is described, then, in section 4 , the model is finally validated with experimental data. 


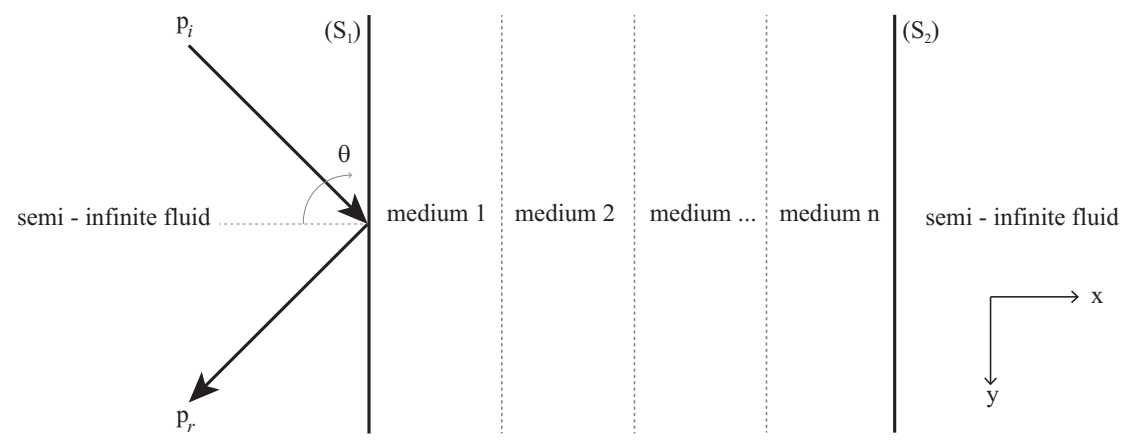

Figure 1: An acoustic wave impinging on a stratified structure with a propagation angle $\theta$. The multilayer element is surrounded by a semi-infinite fluid on both sides.

\section{Numerical model and theoretical background}

\subsection{Transfer matrix method background theory}

The model implemented to compute the sound transmission through the ETICS system is based on the framework of the transfer matrix method, from now on shortened to TMM. It represents one of the most efficient methods used to describe the acoustic wave propagation through layered structures $[13,14]$. The TMM solves a two-dimensional problem of a plane acoustic wave impinging on the surface $\left(S_{1}\right)$ of the layered element, as sketched in Figure 1. The general formalism of TMM can be expressed as:

$$
V_{\left(S_{1}\right)}=[T] V_{\left(S_{2}\right)}
$$

The vector $V_{\left(S_{1}\right)}$ represents all the variables needed to define the acoustic field on the surface $\left(S_{1}\right)$, while the vector $V_{\left(S_{2}\right)}$ contains the descriptors related to each single layer and all the variables that define the acoustic fields on the surface $\left(S_{2}\right)$, such as pressure, velocity and stresses. The transfer matrix $[T]$ describes the sound transmission through the stratified structure. The size of this matrix depends on the nature of each layer, such as solid, fluid, or poroelastic. The media in the TMM basic formulation are assumed to be laterally infinite, therefore discrepancies between measured and predicted results might be significant, especially in the low frequency range, due to the presence of structural modes in finite size elements and due to the diffraction effect, caused by the finite dimension of the tested panel. The diffraction effect of the finite size element was taken into account by introducing a geometrical radiation efficiency $\sigma_{\text {finite }}$. It was computed using the reduced-order integral formulation provided by Bonfiglio et al. [15], which reduces the computational cost compared to other techniques, such as the spatial windowing technique developed by Villot [16], or the formulation proposed by Rahzi [17]. Although, it was strictly developed for square plates, it proved to give reliable results as long as the plate aspect ratio does not exceed 2:1. Assuming the multilayer element is extended by a semi-infinite fluid on both sides, it is possible to express the relationship between the complex transmission $T$ and complex reflection coefficient $R$ as:

$$
T=-(1+R) \frac{\left[D_{N+1}\right]}{\left[D_{1}\right]}
$$


The complex reflection coefficient is defined as:

$$
R=\frac{Z_{S} \cos \theta-Z_{0}}{Z_{S} \cos \theta+Z_{0}}
$$

where $Z_{0}=\rho_{0} c_{0}$ represent the fluid's characteristic impedance, which is a function of the fluid density $\rho_{0}$ and the speed of sound $c_{0}$, while the surface impedance $Z_{S}$ of the stratified element can be computed as:

$$
Z_{S}=-\frac{\operatorname{det}\left[D_{1}\right]}{\operatorname{det}\left[D_{2}\right]}
$$

An alternative procedure to determine the complex transmission coefficient $T$ and the surface impedance $Z_{s}$, for each frequency and each angle of incidence, which does not involve the calculation of the determinants, is given in Eq. (11.97) and (11.98) of ref. [14]. The global transfer matrix D to model the ETICS system, having $(N+1)$ rows and $(N+2)$ columns, is obtained assuming a semi-infinite fluid termination:

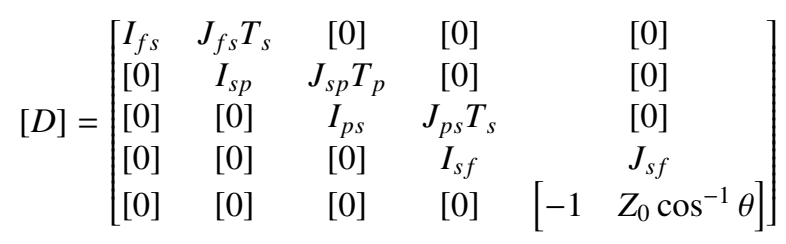

where $I_{i j}$ and $J_{i j}$ are the matrices that couple the different media. The transfer matrices $T_{s}$ and $T_{p}$ describe the sound propagation respectively through elastic solids and poroelastic layers. The exact formulation of these matrices can be found in ref. [14]. The square matrices $\left[D_{1}\right],\left[D_{2}\right]$ and $\left[D_{N+1}\right]$ are obtained by eliminating from $\mathbf{D}$ the first, the second and the $(N+1)^{\text {th }}$ column respectively. For a given propagation angle $\theta$ of the incident wave, the transmission coefficient is defined as:

$$
\tau_{\infty}(\theta)=|T(\theta)|^{2}
$$

The transmission coefficient that takes into account the finite size effect $\tau_{\text {finite }}$ can be determined as:

$$
\tau_{\text {finite }}(\theta)=\tau_{\infty}(\theta) \sigma_{\text {finite }}(\theta) \cos \theta
$$

where the radiation efficiency $\sigma_{\text {finite }}$ represents the previously mentioned finite size correction for a given angle of incidence $\theta$. In the implemented code the transmission coefficient is computed for a frequency vector which contains nine frequencies for each one-third octave band. The transmission coefficient for each one-third octave is obtained by averaging the nine values that fall within the frequency band.

\subsection{Sound bridges correction}

So far only the multilayer structure without structural bridges has been considered. To take into account the mechanical bridges in the ETICS system, Vigran's decoupled approach [6] was conveniently modified. The basic assumption of the decoupled approach is to consider the transmission through the different media and the transmission through the bridges as two independent and additive paths. The total transmission loss $T L_{t o t}$ is determined by adding a correction term to the transmission coefficient $\tau_{\text {finite }}$ obtained from the TMM algorithm. The correction term is calculated as the ratio between the power radiated only due to the bridges action $W_{b}$ over the sound 
power $W_{a}$ that would be radiated by the structure, without mechanical connections, due to the airborne excitation. For each propagation angle of the incident sound field, the total transmission coefficient $\tau_{\text {tot }}$ is given by:

$$
\tau_{\text {tot }}=\tau_{\text {finite }}\left(1+\frac{W_{b}}{W_{a}}\right)
$$

The diffuse field excitation transmission loss $T L_{t o t, d}$ is determined as:

$$
T L_{t o t, d}=-10 \log \frac{\int_{\theta_{\min }}^{\theta_{\max }} \tau_{t o t}(\theta) \cos \theta \sin \theta d \theta}{\int_{\theta_{\min }}^{\theta_{\max }} \cos \theta \sin \theta d \theta}
$$

where the angles $\theta_{\min }$ and $\theta_{\max }$ represent the propagation limits of incident sound wave. In the implemented algorithm the finite range of angles $0 \leq \theta \leq 90^{\circ}$ was used. The sound power ratio, introduced in Eq. (8) to take into account the transmission through the structural bridges, can be computed as:

$$
\frac{W_{b}}{W_{a}}=\frac{\sigma_{b}}{\sigma_{a}}\left\langle\left|\frac{v_{S_{2}, b}}{v_{S_{1}, F}}\right|^{2}\right\rangle\left\langle\left|\frac{v_{S_{1}, F}}{v_{S_{1}}}\right|^{2}\right\rangle\left\langle\left|\frac{v_{S_{1}}}{v_{S_{2}}}\right|^{2}\right\rangle
$$

The first term in the right-hand side of Eq. (10), is the ratio between the radiation efficiency of the plaster layer driven by one of the bridges $\sigma_{b}$, over the airborne radiation efficiency of the same element excited by an acoustic sound field $\sigma_{a}$. Analogously, $v_{S_{2}, b}$ is the mean square vibration velocity of the surface $S_{2}$ driven by the mechanical bridge, while $v_{S_{1}}$ and $v_{S_{2}}$ are the mean square velocities averaged over the surface $\left(S_{1}\right)$ and $\left(S_{2}\right)$ respectively, without the structural connections. The force exerted by the bridges depends on the vibration velocity of surface they are connected with, represented in this model by $v_{S_{1}, F}$, as shown in Figure 3 . While it is a perfectly reasonable thin plate approximation, the mean square velocity cannot be assumed equal over the two opposite surfaces of a thick plate: $v_{S_{1}, F} \neq v_{S_{1}}$.

The radiation efficiency $\sigma_{b}$ has been defined, in Vigran's model, as the link between the sound power radiated due to the action of one bridge and the surface mean square velocity of the restricted area over which the bridge is acting. However, this approach is not suitable for the ETICS system, since the bridge-driving action has a global influence on the velocity over the thin finishing layer surface. In fact, the presence of structural connections does not increase the velocity only locally, over a restricted area the bridge acts on, but it affects the entire radiating surface. In Figure 2 the measured velocity levels are mapped over a one-square-meter sample of the studied structure, described in the next section. Although, the structural connections are well detectable in Figure 2(a), their action affects the vibration velocity over the entire surface compared to the element without mechanical connections in Figure 2(b). The presence of structural connections influence the whole radiating surface, although the highest levels coincide with the bridges, also the velocity in the bays is significantly increased. The radiation efficiency $\sigma_{b}$ of the mechanically excited plate is determined according to Davy's equations presented in ref. [18], which take into account both the resonant and the near field contribution:

$$
\sigma_{b}=\left\{\begin{array}{cc}
\sigma_{r}\left(1+\frac{1}{r}\right) & \text { if } f<f_{c} \\
\sigma_{r} & \text { if } f \geq f_{c}
\end{array}\right.
$$



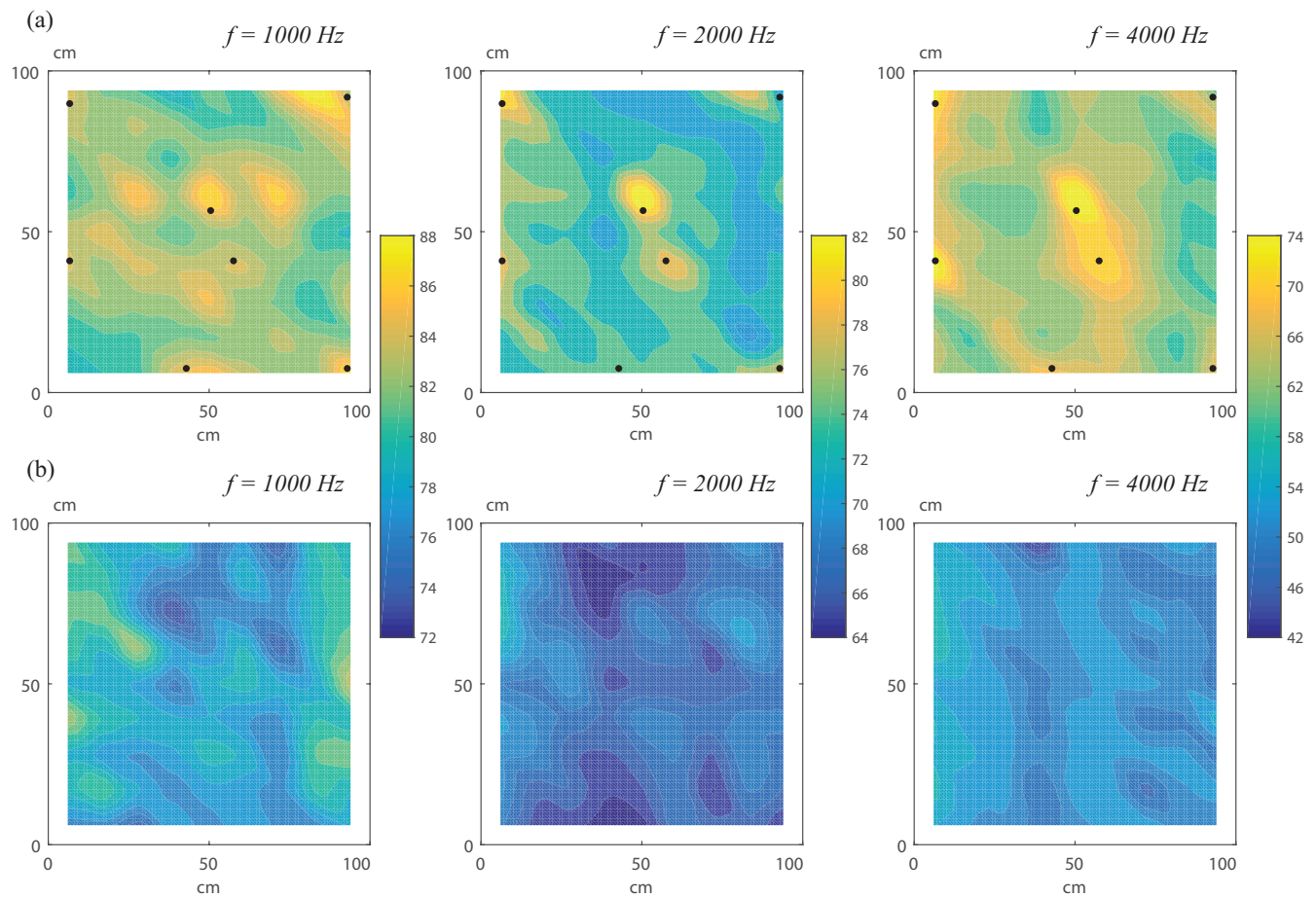

Figure 2: Mapping of the vibration velocity levels distribution on a 1 square meter sample of the finishing layer of the ETICS system. (a) Mechanical connection mounting condition: $\bullet 7$ bridges per square meter represents the standard in real conditions. (b) Mounting condition without mechanical connections.

where $\sigma_{r}$ is the radiation efficiency of the resonant vibration field of the panel. This acoustic descriptor is commonly used when a mechanical broadband excitation is involved, assuming: (i) a high modal density and modal overlap over the entire frequency range; (ii) that the sound power is only radiated by resonant uncorrelated modes; (iii) that all the modes within the frequency band have the same vibrational energy. In the same paper Davy presented the average radiation efficiency for a rectangular plate excited by diffuse sound field.

$$
\sigma_{a}=\left\{\begin{array}{cc}
\frac{r \sigma_{r}+\sigma_{n r}}{1+r} & \text { if } f<f_{c} \\
\sigma_{r} & \text { if } f \geq f_{c}
\end{array}\right.
$$

where $\sigma_{n r}$ is the radiation efficiency of the non-resonant vibrational field excited by an incident diffuse airborne sound. The term $r$ is the ratio of the power radiated by the resonant vibrational field to the power radiated by the vibrational near field, for the point-excited panel. The term $r$ is also the ratio of the resonant vibrational energy to the non-resonant vibrational energy for the panel acoustically excited by a diffuse sound field. In both the cases this ratio is given by:

$$
r=\frac{\pi \omega_{c} \sigma_{r}}{4 \omega \eta}
$$

where $\omega$ is the angular frequency, $\omega_{c}$ the critical angular frequency and $\eta$ represents the loss factor 
of the considered plate. Above the critical frequency $f_{c}$ it is not possible to distinguish between the resonant and non-resonant vibrational fields. Thus in this frequency range $\sigma_{r}=\sigma_{b}=\sigma_{a}$ and the radiation efficiency of the plate tends asymptotically to unity as the frequency increases.

The mean square velocity of the finishing plate, mechanically excited by a number of pointbridges per square metre $n_{S}$, can be computed according to ref.[11] as:

$$
\left\langle\left|v_{S_{2}, b}\right|^{2}\right\rangle=n_{S} \frac{\left|F_{b}\right|^{2}}{\omega \eta_{S_{2}} m_{S_{2}}} \operatorname{Re}\left(Y_{S_{2}}\right)
$$

where $m_{S_{2}}=\rho_{S_{2}} h_{S_{2}}$ is the mass per unit area of the plaster layer, being $\rho_{S_{2}}$ its density and $h_{S_{2}}$ its thickness. The point-drive mobility $Y_{S_{2}}$ for a thin plate is given by:

$$
Y_{i}=\frac{\omega_{c, i}}{8 m_{i} c_{0}^{2}}
$$

The exciting force $F_{b}$ is determined as a function of the vibration velocity of the basic wall excited by the diffuse sound field as:

$$
\left|F_{b}\right|^{2}=\frac{\left\langle\left|v_{S_{1}, F}\right|^{2}\right\rangle}{\left|Y_{S_{1}}+Y_{S_{2}}\right|^{2}}
$$

Under the thin plate theory the vibration velocity on the opposite surfaces of a plate is assumed to be equal. However, as the thin plate theory is limited in frequency range, for a massive structure such as the basic wall in the ETCIS system, this might not be verified in the entire frequency range. Therefore, the point-exciting force is derived from the vibration velocity of the surface the bridges are connected with, $v_{S_{1}, F}$, namely the velocity of the inner side surface of the basic wall, on the interface with the mineral wool layer, as shown in Figure 3. In literature different criteria for the thin plate theory limit can be found, for example the bending wavelength being greater than 6 times the plate thickness. In the frequency range where the thin plate theory is not valid the point mobility of the basic plate $Y_{S_{1}}$ should be derived from the equation developed for thick plates in [5]. On the other hand such point mobility is very small compared to the mobility of the plaster layer $Y_{S_{2}}$ and can be approximated as $Y_{S_{1}}=0$. The second term of Eq. (10) is thus given by:

$$
\left\langle\left|\frac{v_{S_{2}, b}}{v_{S_{1}, F}}\right|^{2}\right\rangle=\frac{n_{S}}{\omega \eta_{S_{2}} m_{S_{2}}\left|Y_{S_{2}}\right|}
$$

It should be mentioned, that since within the TMM the layers are infinitely extended, the reflections of the transverse wave velocity at the edges are not included, Therefore, below half the critical frequency of the basic wall the velocity derived from the TMM represents the forced vibrational response rather than the resonant one. For this reason, below the coincidence, a factor $Q$, given in Eq. (18) of ref. [11] as a function of the ratio between the resonant and the forced vibrational energy, needs to be included in the numerator of Eq. (17). However, considering that the critical frequency of the basic wall falls between the $160 \mathrm{~Hz}$ and $200 \mathrm{~Hz}$ bands, where the bridges' action is not significant, it has been neglected.

The surfaces velocity ratio $\left|v_{S_{1}} / v_{S_{2}}\right|^{2}$, like in Vigran's model, is derived from the transfer matrix computed for the multilayer structure in absence of mechanical bridges. The main differences from Vigran's approach is the possibility to consider many kinds of layers, and matrices 


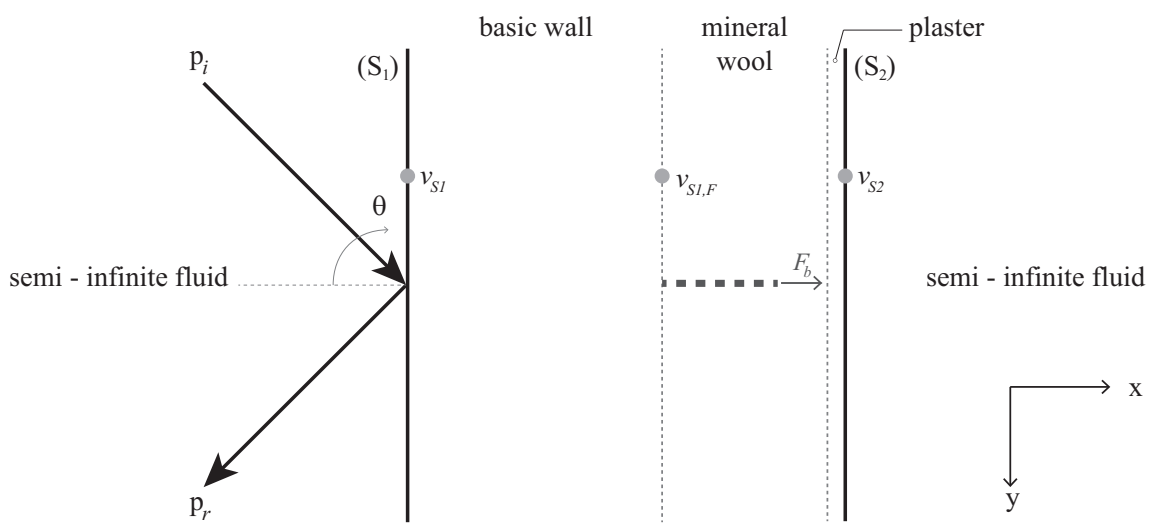

Figure 3: Diagram of the multilayer ETICS system modelled using TMM. In order to consider the transmission contribution due to the bridges action, which is neglected by the TMM, the mechanical force $F_{b}$ determined as a function of the velocity $v_{S_{1}, F}$.

of any size. Vigran's model was developed only for thin plates and low-density fibrous/porous materials, which can be described with good approximation as an equivalent fluid using the laws of Delany and Bazley, depending only on their air flow resistivity and thickness. In other words, only a [2×2] transfer matrix is allowed and no coupling matrices are needed. However, when thick plates or poroelastic layers are modelled, matrices of different size are involved, thus coupling matrices are needed. In this case, the derivation of the ratio between surfaces' mean square velocities, provided in Vigran's model, cannot be applied, due to the increased size of the global transfer matrix. Therefore, a new procedure to numerically determine $v_{S_{1}}$ and $v_{S_{2}}$ is proposed. As already mentioned when Eq. (1) was introduced, the column vector $V_{\left(S_{1}\right)}$ contains all the variables to completely define the acoustic field in the fluid medium adjoining the surface $\left(S_{1}\right)$, namely the sound pressure and the particle velocity along the $x$-direction:

$$
V_{\left(S_{1}\right)}=\left[p_{f}\left(S_{1}\right), v_{f, x}\left(S_{1}\right)\right]
$$

Since the exciting acoustic pressure is arbitrary, it is possible to delete the first column of the transfer matrix $\mathbf{D}$, given in Eq. (5), obtaining a square matrix, indicated as $\left[D_{1}\right]$. The column vector $\bar{V}_{\left(S_{2}\right)}$, containing all the variables that describe the acoustic field in the fluid domain adjoining the surface $\left(S_{2}\right)$, other than the variables that define the transmission through the different layers, can be computed by solving the algebraic system:

$$
\left[D_{1}\right] \bar{V}_{\left(S_{2}\right)}=P
$$

where the vector $P$ is the first column deleted from the matrix $\mathbf{D}$ multiplied by -1 . Due to the continuity condition, the vibration velocity over the external surfaces is equal to the particle velocity of the adjoining fluid media. Thus, for each propagation angle $\theta$ the complex velocities 
Table 1: Elastic properties of the equivalent homogeneous plate used as input data for the basic wall in the TMM model

\begin{tabular}{|c|c|c|c|c|c|}
\hline$f[\mathrm{~Hz}]$ & $E[\mathrm{~Pa}]$ & $f[\mathrm{~Hz}]$ & $E[\mathrm{~Pa}]$ & $f[\mathrm{~Hz}]$ & $E[\mathrm{~Pa}]$ \\
\hline 100 & $2.13 \cdot 10^{10}$ & 400 & $6.68 \cdot 10^{9}$ & 1600 & $2.58 \cdot 10^{9}$ \\
\hline 125 & $1.77 \cdot 10^{10}$ & 500 & $5.54 \cdot 10^{9}$ & 2000 & $2.58 \cdot 10^{9}$ \\
\hline 160 & $1.44 \cdot 10^{10}$ & 630 & $4.57 \cdot 10^{9}$ & 2500 & $2.58 \cdot 10^{9}$ \\
\hline 200 & $1.19 \cdot 10^{10}$ & 800 & $3.74 \cdot 10^{9}$ & 3150 & $2.58 \cdot 10^{9}$ \\
\hline 250 & $9.89 \cdot 10^{9}$ & 1000 & $3.10 \cdot 10^{9}$ & 4000 & $2.58 \cdot 10^{9}$ \\
\hline 315 & $8.15 \cdot 10^{9}$ & 1250 & $2.58 \cdot 10^{9}$ & 5000 & $2.58 \cdot 10^{9}$ \\
\hline
\end{tabular}

$v_{S_{1}, \theta}$ and $v_{S_{2}, \theta}$ are determined from the vector $\bar{V}_{\left(S_{2}\right)}$ as the first and last element respectively:

$$
\bar{V}_{\left(S_{2}\right)}=\left\{\begin{array}{c}
v_{S_{1}, \theta} \\
\vdots \\
v_{S_{1}, F, \theta} \\
\vdots \\
v_{S_{2}, \theta}
\end{array}\right\}
$$

The same procedure also allows one to obtain the velocity $v_{S_{1}, F}$, that the mechanical force $F_{b}$ depends upon. Its position in the vector $\bar{V}_{\left(S_{2}\right)}$ depends on the type of layers and their arrangement in the TMM model. In the model presented here, $v_{S_{1}, F}$ is the transverse velocity of basic wall, defined as a solid elastic layer and is the third element of the column vector $\bar{V}_{\left(S_{2}\right)}$. The derivation of $v_{S_{1}, F}$ is computationally costless. In the case that the thin plate theory is valid over the entire frequency range $v_{S_{1}, F}=v_{S_{1}}$ and the third ratio on the right-hand side of Eq. (10) would be equal to 1 .

\section{Investigated structure}

Experimental measurements have been performed in the wall sound insulation test facility of the University of Ferrara in order to investigate the reliability of the model and validate the assumptions made for its derivation. The studied structure is a massive wall clad with the ETICS system. The basic partition is a masonry wall plastered on both sides. Its total thickness is equal to $150 \mathrm{~mm}$. Masonry walls are highly non-homogeneous and anisotropic structures and it is not easy to determine the elastic properties needed as input data in prediction calculations. To simplify the problem, it was modelled as an equivalent homogeneous solid layer, described by frequency dependent elastic properties, instead of elastic constants, as is common when using homogenization techniques [19]. The frequency dependent elastic modulus, reported in Table 1 was derived from the measured transmission loss, using a minimization algorithm, developed specifically within the TMM framework, as described in ref. [20], assuming the Poisson's ratio $v=0.33$ and a constant loss factor $\eta=0.045$ as typical values for this kind of partition. The basic wall was clad with mineral wool slabs $100 \mathrm{~mm}$ thick, finished with $5 \mathrm{~mm}$ of reinforced cement plaster. The high density mineral wool layer is modelled as a poroelastic medium to consider both the fluid and the solid phase. The five parameters related to the fluid phase, namely airflow 


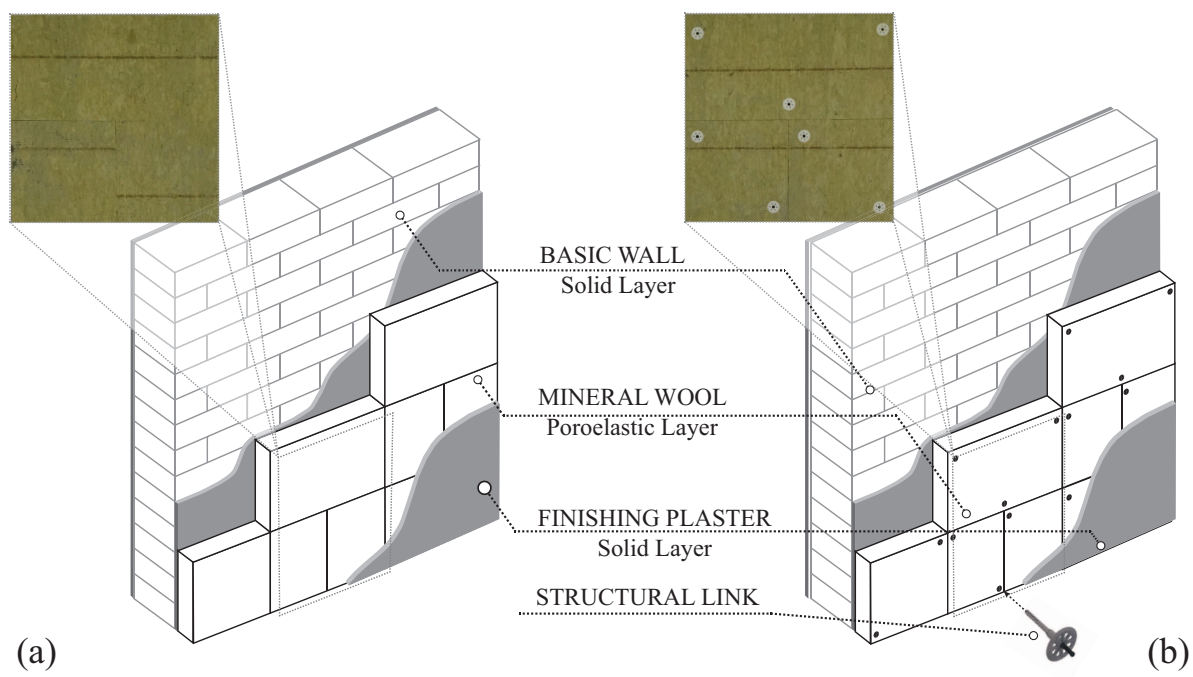

Figure 4: Diagram of the ETICS system mounted on the basic masonry wall and the definition of TMM media. (a) The mounting condition involves only adhesive mortar without mechanical connections; (b) Both mortar and mechanical fixings are used to mount the cladding system on the basic wall.

resistivity $\sigma$, open porosity $\phi$, tortuosity $\alpha$, viscous and thermal characteristic lengths $\Lambda$ and $\Lambda^{\prime}$, were experimentally determined using laboratory measurements combined with an inversion procedure algorithm [21]. The mechanical parameters of the solid skeleton were determined from quasi-static measurements [22]. The cement plaster layer, like the basic wall, was modelled as an elastic solid medium using input values obtained from the literature. A diagram of the multilayer element modelled with the TMM, for both the tested mounting conditions, is shown in Figure 4, and the input data used for each single medium are given in Table 2.

In the first configuration the mineral wool slabs were fixed to the basic wall using only adhesive mortar, applied over approximately $40 \%$ of the surface. It was necessary to implement this configuration in order to determine experimentally the transmission loss provided by the ETICS system, without the additional structure-borne transmission path via the mechanical bridges. However due to static reasons, in the practical situation the adhesive fixing is not enough. It is necessary to also provide mechanical connections. In this second configuration, the structure was mounted by applying 7 mechanical bridges per square meter, in order to reproduce the in-situ conditions.

\section{Validation measurements and results}

The sound insulation provided by the structure was determined according to the standard ISO EN 10140-2 [23]. The influence of the mechanical connections on the transmission loss provided by the multilayer element was thus, experimentally investigated by comparing the values measured with the two different mounting conditions. This comparison, given in Figure 5, shows clearly that they have a significant influence in the frequency range above the $630 \mathrm{~Hz}$ band, where the two experimental curves start to split apart, and a reduction of more than $10 \mathrm{~dB}$ 
Table 2: Mechanical, geometrical and acoustic descriptors used as input data in the TMM model

\begin{tabular}{cccc}
\hline & Eq. homogeneous plate & Mineral wool slabs & Reinforced plaster \\
\hline$h[\mathrm{~mm}]$ & 150 & 100 & 5 \\
\hline$\rho\left[\mathrm{kg} / \mathrm{m}^{3}\right]$ & 1770 & 78 & 1500 \\
\hline$E[\mathrm{~Pa}]$ & freq. dependent Tab. 1 & $5.7 \cdot 10^{5}$ & $5 \cdot 10^{9}$ \\
\hline$\eta[-]$ & 0.045 & 0.05 & 0.01 \\
\hline$v[-]$ & 0.33 & 0.01 & 0.3 \\
\hline$\sigma\left[s P a / \mathrm{m}^{2}\right]$ & - & 59100 & - \\
\hline$\phi[-]$ & - & 0.90 & - \\
\hline$\alpha[-]$ & - & 1.00 & - \\
\hline$\Lambda[\mu \mathrm{m}]$ & - & 18.0 & - \\
\hline$\Lambda^{\prime}[\mu \mathrm{m}]$ & - & 36.0 & - \\
\hline
\end{tabular}

can be found. It should be noted that the reduction of transmission loss due to bridges action is much lower than the increase of velocity levels shown in Figure 2. Since the critical frequency of the finishing layer is around $6750 \mathrm{~Hz}$, this can be related to the vibrational resonant field, that is unable to efficiently radiate sound energy away from the plate. In Figure 5, the transmission loss provided by the basic wall is also plotted, only to highlight the contribution of the lining, but it is not part of the investigation. The suitability of the TMM to predict the transmission loss of these stratified building elements was proved by comparing the numerical results with experimental data measured on the structure without mechanical connections. As shown in Figure 6, the TMM gives a good approximation of the transmission loss provided by the element. The predicted curve matches reasonably well with the measured transmission loss over the entire frequency range. The mass-spring-mass frequency is also correctly detected within the $125 \mathrm{~Hz}$ band. In order to investigate the reliability of the model presented here and validate the assumptions made, each term of Eq. (10), used to evaluate the correction factor, is compared with the experimental data and finally the global validity of the model is discussed.

In order to determine experimentally the velocities ratio in absence of structural bridges, the surface vibration velocity was measured, both on the source $v_{S_{1}, \exp }$ and the receiving side $v_{S_{2}, \text { exp }}$, using PCB accelerometers, with a sufficiently low mass $(m \leq 10 \mathrm{~g})$, to avoid any possible influence on the dynamic response of the finishing layer. The vibration velocity over the basic wall surface was averaged over a random distribution of accelerometer positions according to the standard ISO 10848-1 [24]. The velocity on the reinforced plaster surface, which in the experimental configuration was on the receiving side, was averaged over a grid of accelerometer positions, with an equal spacing of $100 \mathrm{~mm}$. This procedure was extremely time consuming but allowed the investigation of the regions over which the structural bridges were acting. In Figure 7, the ratio between the average velocity on the surfaces $\left(S_{1}\right)$ and $\left(S_{2}\right)$, evaluated numerically from the TMM, is compared with the data measured on the structure without connections. While a very good agreement is found in the low frequency range, at higher frequencies the model seems 


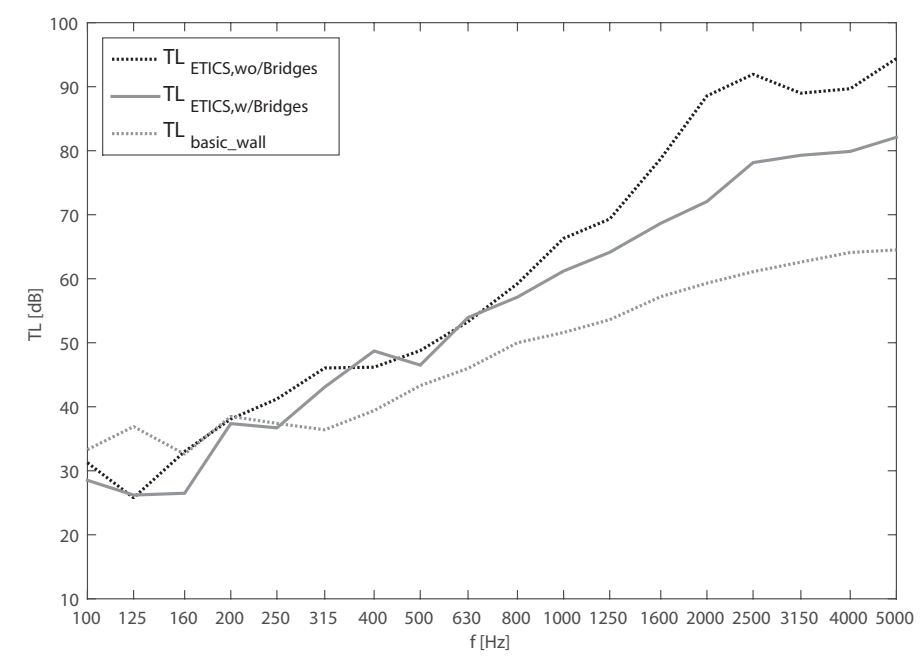

Figure 5: Transmission loss experimentally evaluated on the basic wall and on the multilayer structure, with and without the mechanical fixing.

to overestimate this ratio. The plastered masonry brick wall has been modelled by frequencydependent elastic properties of an equivalent homogeneous layer. This approximation may have an influence on the velocity ratio. However, since the same model provided accurate results in terms of transmission loss, as shown in Figure 6, the discrepancies might be due to experimental uncertainties. In all probability the number of points used to measure the vibration velocity over the surface on the exciting side $\left(S_{1}\right)$, chosen in order to fulfil the distance requirements in ISO 10848, was not enough to obtain an accurate spatial sampling. Therefore, the mean velocity $v_{S_{1}}$ represents an underestimation of the mean velocity of the surface $\left(S_{1}\right)$, as was also found in a recent study on the experimental evaluation of the quantities necessary to define the radiation efficiency [25].

Due to practical reasons it was not possible to measure the mean square velocity on the thin finishing layer driven only by the bridges actions. This is because the reinforced plaster layer cannot be mounted detached from the mineral wool, with a vacuum instead of air in the voids of the mineral wool and only suspended on the bridges. Under the decoupled approach the total radiated power is assumed to be the sum of the airborne radiated power and the sound power radiated due to the bridges action. This relationship can be expressed in terms of radiation efficiencies and mean square velocity as:

$$
\left\langle\left|\frac{v_{2, t o t}}{v_{S_{1}}}\right|^{2}\right\rangle \sigma_{t o t}=\left\langle\left|\frac{v_{S_{2}, b}}{v_{S_{1}}}\right|^{2}\right\rangle \sigma_{b}+\left\langle\left|\frac{v_{S_{2}}}{v_{S_{1}}}\right|^{2}\right\rangle \sigma_{a}
$$

In order to validate the the approach used to calculate the surface velocities ratio of the structure with mechanical connections, the term on the left-hand side of Eq. (21) was experimentally determined and compared with the terms on the right-hand side of the equation numerically computed, in Figure 8. The comparison shows a rather good agreement between the experimental total velocity ratio and the numerical results, which means that the model well approximates the 


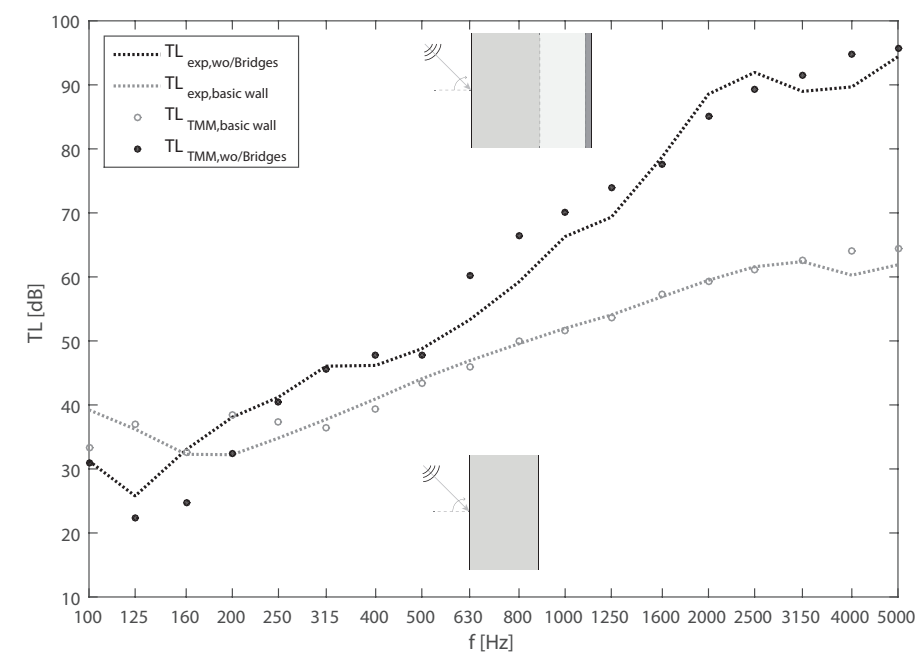

Figure 6: Transmission loss of the ETICS system without structural connection. Comparison between the TMM results and experimental data.

trend of the vibration velocity of the finishing layer driven by the structural bridges.

The same practical issue determined also the impossibility to experimentally evaluate the ratio of the radiation efficiencies. Nevertheless, some considerations on the radiated sound power can be made in order to validate the hypothesis of the model. The sound power radiated by the finishing thin plate, in both the mounting conditions, was determined through sound intensity measurements, performed according to the standard ISO 9614-3 [26]. The lateral walls and the floor were shielded with absorbing material, to reduce the interference given by the reverberant sound field. In Figure 9, the sound power levels radiated from the structure with $W_{\text {tot,bridges }}$ and without mechanical connections $W_{\text {wo/bridges }}$ are compared. Below the $630 \mathrm{~Hz}$ band, no significant differences are founded, while the two curves progressively split apart as the frequency increases. At low frequencies transmission via the mineral wool frame and the air that fills the voids is dominant. However, as the driving action of the structural connections becomes significant the transmission via the bridges becomes more and more important. This is particularly in line with the assumption made in the model, since at low frequency there is no difference between the layered element with or without bridges, but at higher frequencies, approaching the critical condition of the plaster layer, the resonant and the near field radiation, due to the mechanical excitation, becomes the dominant component.

The global reliability of the model was finally verified by comparing the transmission loss obtained from the TMM with the experimental results in Figure 10. The numerical results provided by the proposed model are in rather good agreement with the experimental data and the sound insulation reduction in the high frequency region is well approximated. Although, in the mid-frequency range a slightly higher transmission loss is computed with the TMM, while above $2000 \mathrm{~Hz}$ the reduction caused by the bridges action is overestimated, due to the assumption of infinite stiff structural connections, as alredy discussed in [27]. In the same Figure the transmission loss obtained with Vigran's formulation is plotted as a further proof that the different 


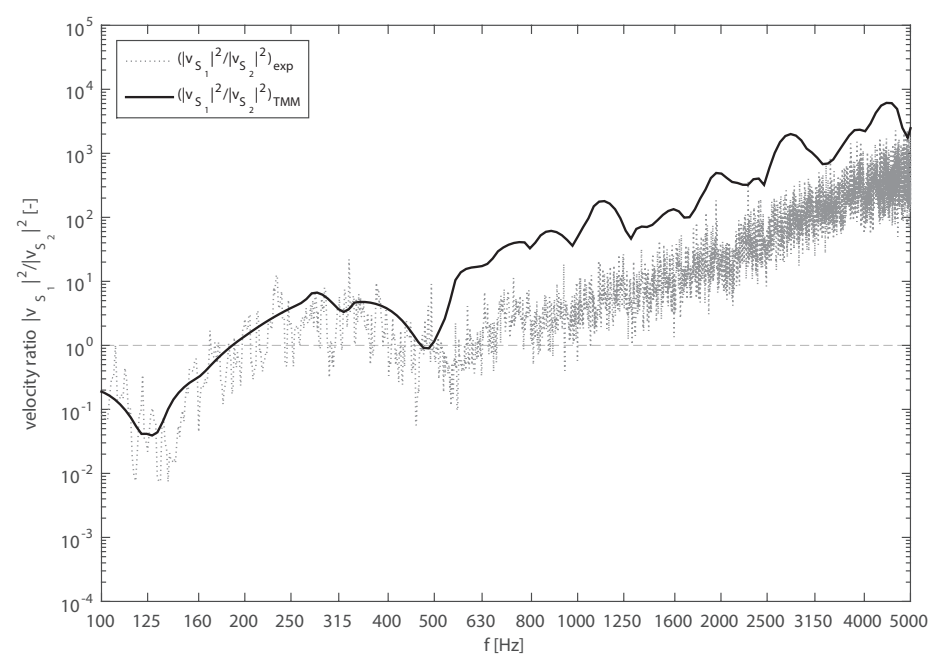

Figure 7: Ratio between the mean square velocities on the external surfaces of the multilayer structure. The experimental results, measured on the sample without mechanical bridges, are compared with the numerical data obtained from the TMM

assumptions made in this model are correct and necessary to consider double wall system consisting of two plates with incomparable thickness and mass, or when the cavity is filled with high density porous or fibrous material that cannot be modelled as an equivalent fluid. Even though Vigran's model works very well with double leaf lightweight structures, it clearly fails in the prediction of the reduction of the ETICS transmission loss, caused by the presence of point-bridges, providing an underestimated correction term. For a better understanding of the influence of structural connections, the differences $\Delta$ TL between the transmission loss without and with bridges, either measured and predicted, are given Figure 10. The TMM provides a good approximation of the experimental TL differences. The discrepancies found at high frequencies are mostly related to the assumption of infinitely stiff bridges, which causes an overestimated reduction of the transmission loss of the system.

\section{Conclusion}

A prediction model, developed to investigate the transmission loss provided by the ETICS cladding for building façades, used to achieve a good thermal and sound insulation performance, has been presented. The basic building wall and the cladding system can be seen as a multilayer structure; therefore, the TMM is a particularly useful tool to describe sound propagation through the different media. Due to static reasons it is necessary to attach the lining slabs using mechanical connections other than an adhesive layer. Although many prediction models can be found in literature to model structural links in stratified elements, or double panel partitions, it has been shown that those are not suitable for the ETICS systems. A new model, based on the decoupled approach has been proposed within the TMM. It takes inspiration from Vigran's formulation, which was conveniently modified in order to make it suitable for such building elements. The structure-borne sound transmission through mechanical fixings is considered by 


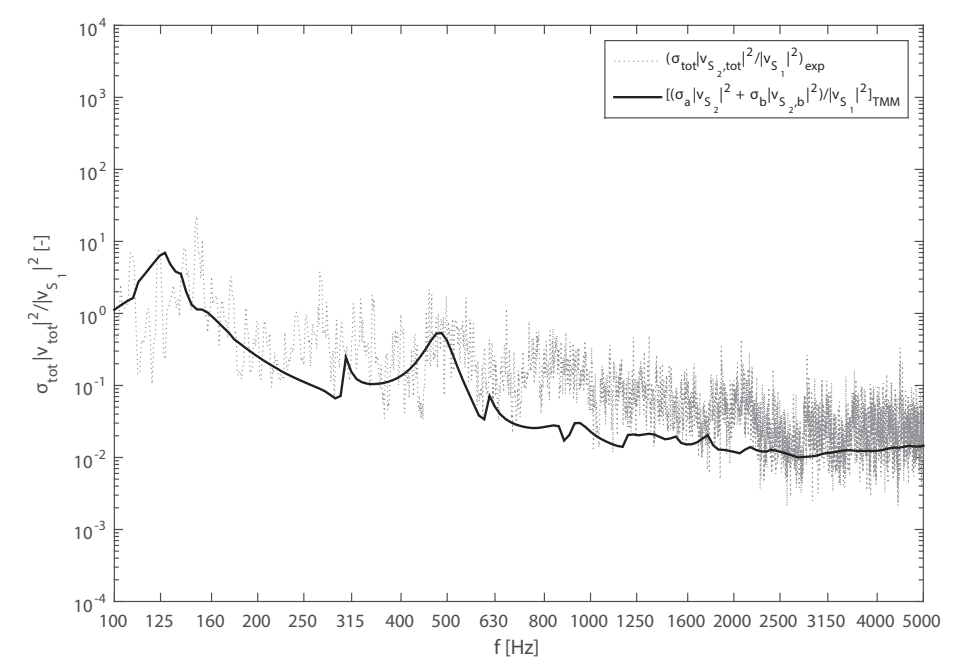

Figure 8: Comparison between the total vibration velocity measured on the plaster layer surface, on the structure with mechanical connections, multiplied by the radiation efficiency experimentally evaluated and the numerical surface velocity ratios with and without bridges multiplied respectively by the airborne and point-driven radiation efficiency.

means of a correction factor added to the transmission coefficient obtained from the TMM model. All the parameters necessary to determine the correction term were accurately described, defining all the assumptions made in their derivation. The validation of each parameter and the global reliability of the model were investigated with experimental measurements. At first the influence of mechanical connections on the sound insulation provided by the element was investigated. Due to the structure-borne transmission, a clear reduction of the sound insulation above the 630 $\mathrm{Hz}$ frequency band is shown. Comparing the transmission loss, measured on the tested element without structural links, with the numerical results obtained from TMM a very good agreement was found, proving that this is a suitable tool for such elements. The validation shows that all the assumptions of the model are quite reasonable and the numerical parameters approximate very well the experimental data. Differently from what has been done in the methods based on the decoupled approach that can be found in literature, the ratio of the mechanical excitation and airborne excitation radiation efficiency of the plate on the receiving side is here considered. The power radiated due the structural connection is thus computed by taking into account the mean square velocity averaged over the entire plate surface, instead of considering only a restricted area over which each mechanical force is exerted. Comparing the maps of velocity distribution measured for the two mounting conditions, it can be seen that the presence of structural connections influence the whole radiating surface, although the highest levels coincide with the bridges, also the velocity in the bays is increased. The numerical evaluation of the surface velocities ratios matches very well the experimental results both for the case with sound bridges and in absence of structural links. Comparing the radiated sound power measured in the two different mounting configurations it was found that there was a similar radiation behaviour at low frequencies, which is governed by transmission via the mineral wool. However, at high frequencies the radiation, due to the bridges action, becomes more and more significant. The global reliability of the model was finally checked by comparing the measured transmission loss with the results obtained from 


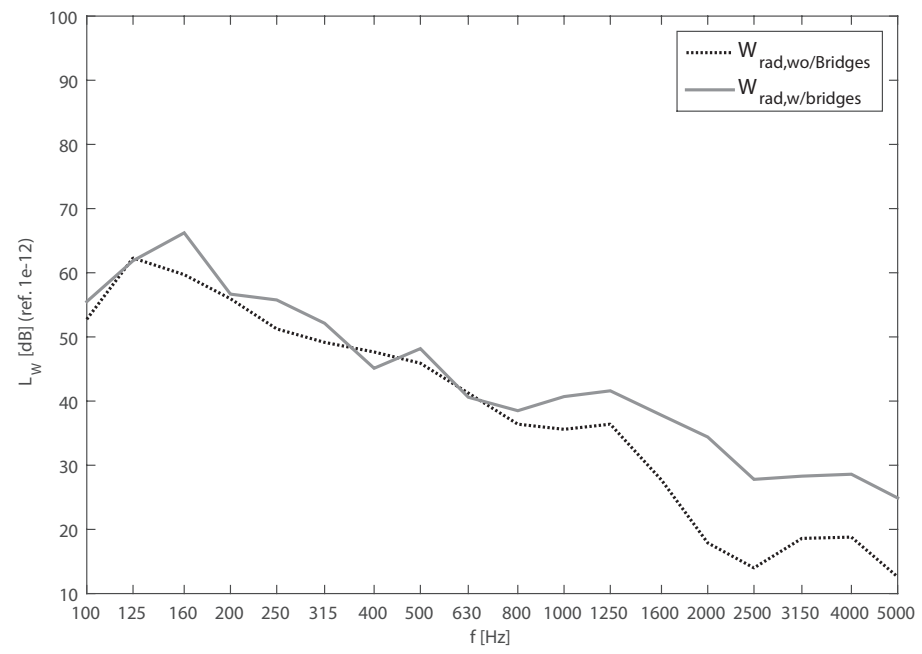

Figure 9: Sound power levels radiated by the building element, experimentally determined from sound intensity measurements.

the TMM. The model provides a good approximation of the experimental sound insulation accurately predicting the frequency region where the sound insulation of the building element is reduced due to the bridges actions.

\section{References}

[1] L. Parati, B. P. Farbood, M. Borghi, May retrofit also include acoustics aspects?, Energy Procedia 78 (2015) 158163.

[2] L. Weber, B. Kaltbeitzel, Schalldämmung von Wrmedmm-Verbundsystemen mit zweilagiger Dämmschicht - Bauforschung Band T 3341 (IBP-Bericht B-BA 1/2016).

[3] V. Hongisto, Sound insulation of double panels-comparison of existing prediction models, Acta Acustica united with Acustica 92 (1) (2006) 61-78.

[4] B. H. Sharp, Prediction methods for the sound transmission of building elements, Noise Control Engineering 11 (2) (1978) 53-63.

[5] L. Cremer, M. Heckl, B. A. T. Petersson, Structure-borne sound, 3rd Edition, Springer-Verlag, Berlin Heidelberg, 2005.

[6] T. E. Vigran, Sound transmission in multilayered structures-introducing finite structural connections in the transfer matrix method, Applied Acoustics 71 (1) (2010) 39-44.

[7] F. Fahy, P. Gardonio, Sound and Structural Vibration. Radiation, Transmission and Response, 2nd Edition, Academic Press in an imprinting of Elsevier, Oxford, UK, 2007.

[8] Q. Gu, J. Wang, Effect of resilient connection on sound transmission loss of metal stud double panel partitions, Chinese Journal of Acoustics 2 (2) (1983) 113-126.

[9] J. L. Davy, A model for predicting the sound transmission loss of walls, in: Proceedings of the Australian Vibration and Noise Conference 1990, Institution of Engineers, Melbourne, Australia, 1990, pp. 23-27.

[10] J. L. Davy, Predicting the sound transmission loss of cavity walls, in: Proceedings of Interior Noise Climates: National Conference of Australian Acoustical Society, Australian Acoustical Society, Perth, Australia, 1990, pp. $1-16$.

[11] J. L. Davy, Sound transmission of cavity walls due to structure borne transmission via point and line connections, The Journal of the Acoustical Society of America 132 (2) (2012) 814-821.

[12] R. Craik, R. Smith, Sound transmission through lightweight parallel plates. Part II: Structure-borne sound, Applied Acoustics 61 (2) (2000) 247-269. 


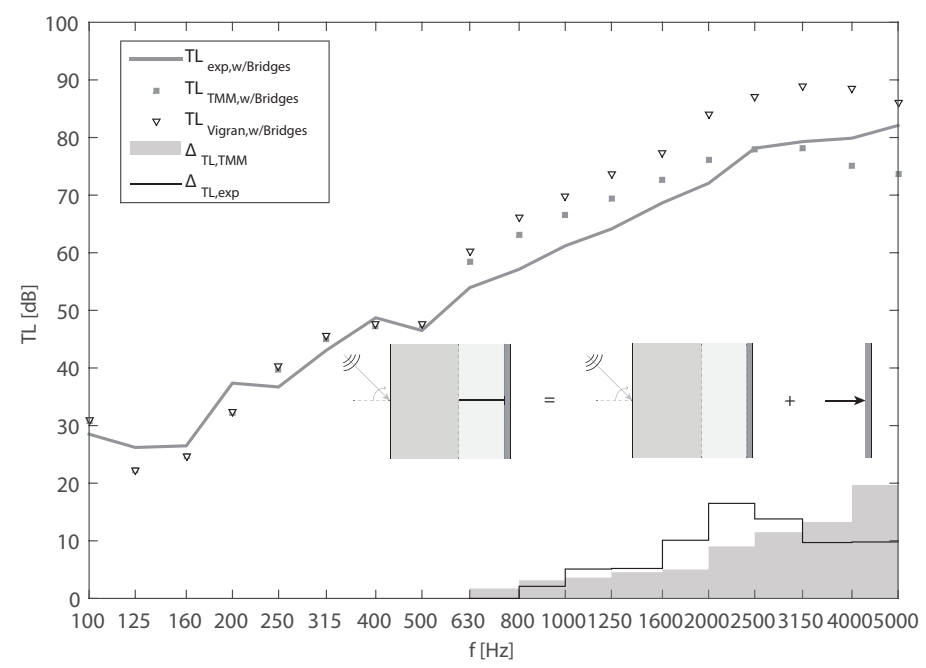

Figure 10: Transmission loss of the ETICS system with structural connection. Comparison between the TMM results and experimental data.

[13] M. Munjal, Response of a multi-layered infinite plate to an oblique plane wave by means of transfer matrices, Journal of Sound and Vibration 162 (2) (1993) 333-343.

[14] J. Allard, N. Atalla, Propagation of sound in porous media: modelling sound absorbing materials, 2nd Edition, John Wiley \& Sons, Ltd, Chichester, UK, 2009.

[15] P. Bonfiglio, F. Pompoli, R. Lionti, A reduced-order integral formulation to account for the finite size effect of isotropic square panels using the transfer matrix method, The Journal of the Acoustical Society of America 139 (4) (2016) 1773-1783.

[16] M. Villot, C. Guigou, L. Gagliardini, Predicting the acoustical radiation of finite size multi-layered structures by applying spatial windowing on infinite structures, Journal of sound and vibration 245 (3) (2001) 433-455.

[17] D. Rhazi, N. Atalla, A simple method to account for size effects in the transfer matrix method, The Journal of the Acoustical Society of America 127 (2) (2010) EL30-EL36.

[18] J. L. Davy, D. J. Larner, R. R. Wareing, J. R. Pearse, The acoustic radiation impedance of a rectangular panel, Building and Environment 92 (1) (2015) 743-755.

[19] D. Backström, A. C. Nilsson, Modelling the vibration of sandwich beams using frequency-dependent parameters, Journal of Sound and Vibration 300 (3) (2007) 589-611.

[20] A. Santoni, P. Bonfiglio, P. Fausti, N. Zuccherini Martello, Sound insulation of heavyweight walls with linings and additional layers: Numerical investigation., in: Proceeding of the $10^{\text {th }}$ European Congress and Exposition on Noise Control Engineering, EAA-NAG-ABAV, Maastricht, Netherlands.

[21] P. Bonfiglio, F. Pompoli, Inversion problems for determining physical parameters of porous materials: Overview and comparison between different methods, Acta Acustica united with Acustica 99 (3) (2013) 341-351.

[22] P. Bonfiglio, F. Pompoli, P. Shravage, Quasi-static evaluation of mechanical properties of poroelastic materials: static and dynamic strain dependence and in vacuum tests, EAA-ASA-SFA, Paris,France.

[23] EN ISO 10140-2 - Acoustics: Laboratory measurement of sound insulation of building elements - Part 2: Measurement of airborne sound insulation, Standard, International Organization for Standardization, Geneva, CH (2010).

[24] ISO 10848-1 -Acoustics - Laboratory Measurement of the flanking transmission of airborne and impact sound between adjoining rooms.-Part 1: Frame Document., Standard, International Organization for Standardization, Geneva, CH (2006).

[25] A. Santoni, P. Bonfiglio, P. Fausti, S. Schoenwald, H.-M. Tröbs, Sound radiation efficiency measurements on cross laminated timber plates, in: Proceedings of the $45^{\text {th }}$ International Congress and Exposition on Noise Control Engineering, Institute of Noise Control Engineering, Hamburg, Germany, 2016, pp. 3697-3707.

[26] ISO 9614-3 -Acoustics - Determination of sound power levels of noise sources using sound intensity-Part 3 Precision method for measurement by scanning, Standard, International Organization for Standardization, Geneva, 
$\mathrm{CH}$ (2002).

[27] T. E. Vigran, Sound insulation of double-leaf walls-allowing for studs of finite stiffness in a transfer matrix scheme, Applied Acoustics 71 (7) (2010) 616-621. 